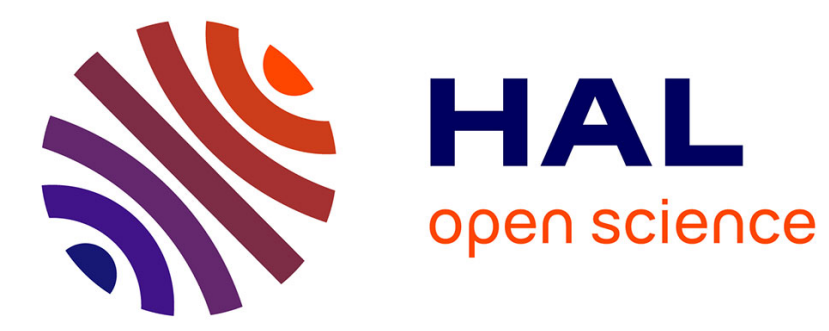

\title{
Elastic energy of a straight dislocation and contribution from core tractions
}

\author{
Emmanuel Clouet
}

\section{To cite this version:}

Emmanuel Clouet. Elastic energy of a straight dislocation and contribution from core tractions. Philosophical Magazine, 2009, 89 (19), pp.1565-1584. 10.1080/14786430902976794 . hal-00400108

\section{HAL Id: hal-00400108 https://hal.science/hal-00400108}

Submitted on 29 Jun 2009

HAL is a multi-disciplinary open access archive for the deposit and dissemination of scientific research documents, whether they are published or not. The documents may come from teaching and research institutions in France or abroad, or from public or private research centers.
L'archive ouverte pluridisciplinaire HAL, est destinée au dépôt et à la diffusion de documents scientifiques de niveau recherche, publiés ou non, émanant des établissements d'enseignement et de recherche français ou étrangers, des laboratoires publics ou privés. 


\title{
RESEARCH ARTICLE
}

\section{Elastic energy of a straight dislocation and contribution from core tractions}

\author{
Emmanuel Clouet ${ }^{\dagger}$ \\ CEA, DEN, Service de Recherches de Métallurgie Physique, \\ F-91191 Gif-sur-Yvette, France \\ (June 29, 2009)
}

\begin{abstract}
We derive an expression of the core traction contribution to the dislocation elastic energy within linear anisotropic elasticity theory using the sextic formalism. With this contribution, the elastic energy is a state variable consistent with the work of the Peach-Koehler forces. This contribution needs also to be considered when extracting from atomic simulations core energies. The core energies thus obtained are real intrinsic dislocation properties: they do not depend on the presence and position of other defects. This is illustrated by calculating core energies of edge dislocation in bcc iron, where we show that dislocations gliding in $\{110\}$ planes are more stable than the ones gliding in $\{112\}$ planes.
\end{abstract}

Keywords: dislocation theory; anisotropic elasticity; core tractions; elastic energy; core energy

\section{Introduction}

One important quantity controlling the physics of dislocations is their elastic energy. It is defined as the integral of the elastic energy density over the whole volume except a small core region surrounding the dislocation line. This excludes the region around the dislocation core where elasticity does not apply because of the too high strains. Using Gauss theorem, the elastic energy can be decomposed in two contributions:

- the one corresponding to an integration along the dislocation cut of the work necessary to create the dislocation. As it is well known, this contribution varies with the logarithm of a characteristic distance of the dislocation microstructure.

- the contribution arising from the work done by the tractions exerted on the tube which isolates the dislocation core. The corresponding contribution to the elastic energy is known as the contribution of the core tractions.

This last contribution is sometimes forgotten. Indeed, the cut contribution is usually the dominant one. Moreover, the core traction contribution disappears when one tries to define the elastic energy of an isolated infinite straight dislocation because of the external cylinder that has to be introduced to prevent the elastic energy from diverging. Nevertheless, if one wants the dislocation elastic energy to be a state variable, i.e. a variable that only depends on the current state and not on the transformation path used to reach this state, both contributions need to be

\footnotetext{
$\dagger$ Email: emmanuel.clouet@cea.fr
} 
taken into account. Bullough and Foreman [1] already showed that the elastic energy of a dislocation loop does not depend on the hypothetical creation mechanism only when both contributions are considered. Lothe and Hirth [2] also noticed that the definition of the elastic energy of a straight dislocation could not be consistent with the work of the Peach-Koehler forces if the contribution of the core tractions was not included in the elastic energy. Finally, Gavazza and Barnett [3] showed that the dislocation elastic energy part associated with the core tractions leads to a contribution to the self-force acting on a dislocation loop.

Despite its importance, no analytical expressions of the core traction contribution to the elastic energy seems to exist in the literature when the elastic anisotropy is considered. The anisotropic linear elasticity theory of dislocations has been developed in the past sixty years. Eshelby, Read and Shockley [4] were the first to express the anisotropic elastic field created by a straight infinite dislocation. Their formalism was then enriched by Stroh [5, 6], leading to what is known as the sextic formalism. Latter, it was shown that the elastic field of a dislocation of arbitrary shape can be obtained from the fields of straight infinite dislocations 7 . But no expression of the contribution of the core tractions to the elastic energy has been obtained until now. The variation of this contribution with a rotation of the dislocation cut is known [8, 9]. It actually corresponds to the angular dependence of the interaction energy between two dislocations derived by Stroh [5]. But an expression of the absolute value of this contribution is not available. Such an analytical expression exists under the assumption that the elastic constants are isotropic [1]. The few studies that considered the elastic anisotropy as well as the contribution of the core tractions 110, 11, 12, 13] calculated this contribution with a numerical integration along the surface of the core cylinder. It is the purpose of this article to obtain an analytical expression of this contribution in the framework of the sextic formalism. Such an expression could be used then in further studies. Potential applications are the extraction from atomic simulations of dislocation energy properties like core energies [8, 9, 14, 15, 16, 17, 18], calculations of dislocation loop self energy [1, 10, 13], or computation of the self-force acting on a dislocation loop [3]. Such an application is presented in this article where core energies of edge dislocation in $\alpha$-iron are determined.

In the first section, we define the elastic energy of a straight dislocation so as to clearly make appear the contribution of the core tractions. We then use isotropic elasticity to illustrate this definition and highlight the importance of this contribution for the coherency of the elastic energy definition. The analytical expression of the core traction contribution within anisotropic linear elasticity is obtained in the following section using the sextic formalism. We finally illustrate the consistency of our results by studying edge dislocations in $\alpha$-iron and calculating their core energies with different simulation methods.

\section{Elastic energy of a straight dislocation}

We assume in this article that the elastic field created by a dislocation can be reduced to the Volterra solution. Eshelby et al. [4] have indeed shown that a straight dislocation in an infinite elastic medium creates in a point defined by its cylindrical

\footnotetext{
${ }^{1}$ For a review, see Ref. 汭.
} 
coordinates $r$ and $\theta$ a displacement given by the Laurent series

$$
\boldsymbol{u}(r, \theta)=\boldsymbol{v} \ln (r)+\boldsymbol{u}_{0}(\theta)+\sum_{n=1}^{\infty} \boldsymbol{u}_{n}(\theta) \frac{1}{r^{n}}
$$

The two first terms of this series, $\boldsymbol{v} \ln (r)+\boldsymbol{u}_{0}(\theta)$ correspond to the Volterra solution: this describes the elastic field of the dislocation far enough from its core. The remaining terms of the series $(n \geq 1)$ are the dislocation core field [19], which arises from non-linearities in the crystal elastic behavior and from perturbations due to the atomic nature of the core. We do not consider this part of the elastic field in our definition of the dislocation elastic energy $\left(\boldsymbol{u}_{n}=0 \forall n \geq 1\right)$ and take only the Volterra solution. The elastic field creates nevertheless tractions on the surface which isolates the dislocation core. As a consequence, a contribution of the core tractions to the dislocation elastic energy exists even when the dislocation core field is neglected. As it will be shown below, this contribution needs to be considered so as to obtain an unambiguous definition of the dislocation elastic energy.

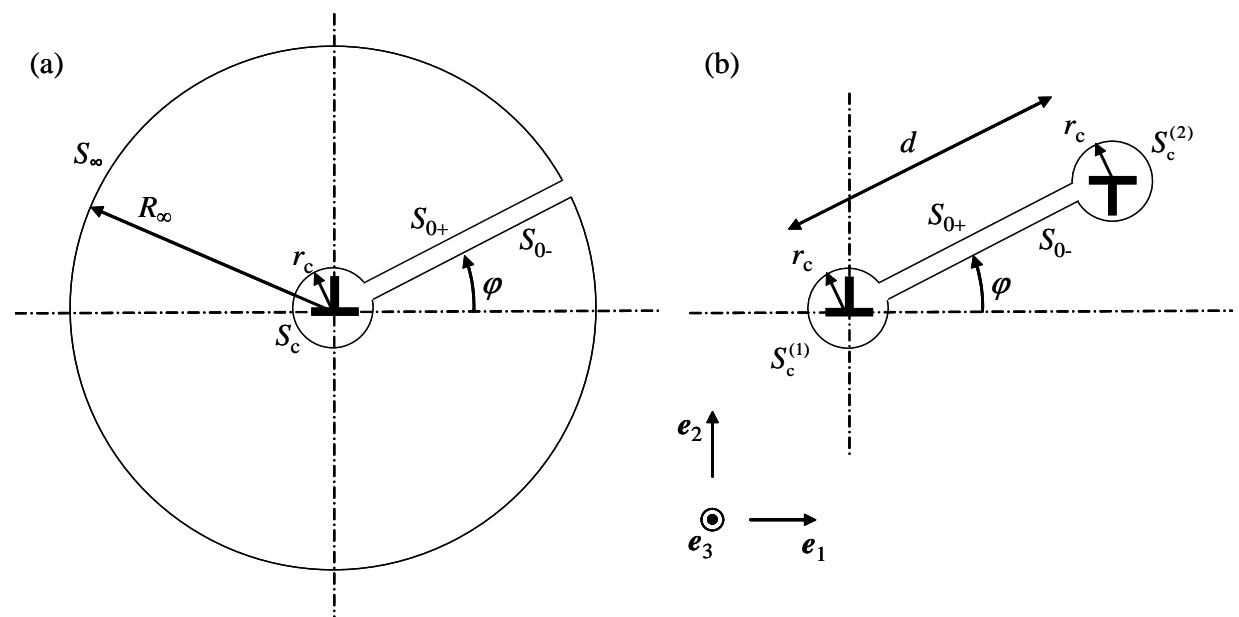

Figure 1. Definition of the contour used to calculate the dislocation elastic energy. (a) Isolated dislocation. (b) Isolated dislocation dipole.

We first consider an isolated dislocation. Two cylinders centered on the dislocation need to be introduced so as to define the elastic energy (Fig. 1 1a). The inner cylinder of surface $S_{\mathrm{c}}$ isolates the dislocation core. Strains are much too high close to the dislocation core to be described by elasticity theory. As a consequence, elastic fields are diverging at the origin and one needs to exclude the core region from the elastic description. As the elastic energy integrated on an infinite volume tends to infinity, one also needs the external cylinder to prevent the elastic energy from diverging. The dislocation elastic energy per unit-length of dislocation is thus defined in the volume $V$ comprised between both cylinders

$$
E_{\text {dislo }}^{\text {elas }}=\frac{1}{2} \iiint_{V} \sigma_{i j} \varepsilon_{i j} \mathrm{~d} V
$$

where $\sigma$ and $\varepsilon$ are respectively the stress and strain created by the dislocation. The use of Gauss theorem allows to transform this volume integral into a surface integral:

$$
E_{\text {dislo }}^{\text {elas }}=\frac{1}{2} \iint_{S} \sigma_{i j} u_{i} \mathrm{~d} S_{j}
$$


where $S$ is a surface enclosing the volume $V$ and isolating any discontinuity of the elastic displacement $\boldsymbol{u}$ generated by the dislocation. A dislocation is by definition the frontier of a surface that has been sheared by the Burgers vector $\boldsymbol{b}$. A displacement discontinuity therefore occurs on the dislocation cut which needs to be isolated by the surfaces $S_{0^{+}}$and $S_{0^{-}}$(Fig. 1 $1 \mathrm{a}$ ). We would like to stress that this cut does not necessary correspond to the dislocation glide plane. The surface appearing in the integral $1 \mathrm{1}$ is then composed of $S_{\mathrm{c}}, S_{0^{-}}, S_{\infty}$, and $S_{0^{+}}$.

The integrals along the surfaces $S_{\mathrm{c}}$ and $S_{\infty}$ cancel because the resultant of forces located in the core is null, and the normals to the surfaces $S_{\mathrm{c}}$ and $S_{\infty}$ have an opposite orientation [5, [6]. As a consequence, the elastic energy of an isolated dislocation integrated between the cylinders of radii $r_{\mathrm{c}}$ and $R_{\infty}$ is limited to the contribution corresponding to the dislocation cut, leading to the the well-known result

$$
E_{\text {dislo }}^{\text {elas }}=\frac{1}{2} b_{i} K_{i j}^{0} b_{j} \ln \left(\frac{R_{\infty}}{r_{\mathrm{c}}}\right)
$$

where the tensor $\mathbf{K}^{0}$ only depends on the elastic constants, and $r_{\mathrm{c}}$ and $R_{\infty}$ are respectively the radii of the inner and external cylinders.

We consider then an isolated dislocation dipole. The first dislocation of Burgers vector $\boldsymbol{b}$ is located at the origin and the second one of Burgers vector $-\boldsymbol{b}$ at the point defined by its cylindrical coordinates $(d, \phi)$ (Fig. [1 b). The two dislocation cuts need to be orientated so that the displacement discontinuity cancel except in the surface bounded by both dislocations. This ensures that no displacement discontinuity occurs far from the dipole and allows the dipole to be mechanically isolated. The elastic energy created by the dipole in the infinite volume is then

$$
E_{\text {dipole }}^{\text {elas }}=\frac{1}{2} \iint_{S}\left(\sigma_{i j}^{(1)}+\sigma_{i j}^{(2)}\right)\left(u_{i}^{(1)}+u_{i}^{(2)}\right) \mathrm{d} S_{j}
$$

where $\boldsymbol{\sigma}^{(1)}$ and $\boldsymbol{\sigma}^{(2)}$ are the stresses created by each dislocation, and $\boldsymbol{u}^{(1)}$ and $\boldsymbol{u}^{(2)}$ the corresponding elastic displacements. The integration surface is composed of the two cylinders $S_{\mathrm{c}}^{(1)}$ and $S_{\mathrm{c}}^{(2)}$ of radii $r_{\mathrm{c}}$ removing the elastic divergence at the dislocation cores, and of the two surfaces $S_{0^{-}}$and $S_{0^{+}}$removing the displacement discontinuity along the dislocation cut (Fig. 1 $1 \mathrm{~b}$ ). One does not need to introduce an external surface like for the isolated dislocation because the elastic energy integrated on the infinite volume now converges.

The integration on both core cylinders leads to the same contribution

$$
E_{\mathrm{c}}^{\mathrm{elas}}(\phi)=\frac{1}{2} \iint_{S_{\mathrm{c}}^{(1)}} \sigma_{i j}^{(1)} u_{i}^{(1)} \mathrm{d} S_{j}=\frac{1}{2} \iint_{S_{\mathrm{c}}^{(2)}} \sigma_{i j}^{(2)} u_{i}^{(2)} \mathrm{d} S_{j} .
$$

The elastic energy of the dislocation dipole is then

$$
E_{\text {dipole }}^{\text {elas }}=2 E_{\mathrm{c}}^{\mathrm{elas}}(\phi)+b_{i} K_{i j}^{0} b_{j} \ln \left(\frac{d}{r_{\mathrm{c}}}\right) \text {. }
$$

We will show in the following that the contribution $E_{\mathrm{c}}^{\text {elas }}$ of the core tractions only depends on the angle $\phi$ defining the azimuthal position of the dislocation dipole. It does not depend on the core radius $r_{\mathrm{c}}$ nor on the separation distance $d$. This contribution is not present in the elastic energy of an isolated dislocation (Eq. 2). This is a consequence of the introduction of an external cylinder to prevent 
the elastic energy from diverging. But an isolated dislocation cannot exist: another defect, like a dislocation with opposite Burgers vector or a surface, is always needed to close the dislocation cut and allows mechanical equilibrium. Therefore, one has to use the "trick" of the external cylinder so as to define the elastic energy of an isolated dislocation. This gives birth to an artefact as the core traction contribution $E_{\mathrm{c}}^{\text {elas }}$ then disappears.

\section{Isotropic elastic media}

We first consider that the elastic constants are isotropic. This allows to obtain simple expressions of the elastic energy which can be easily manipulated so as to illustrate the importance of the core traction contribution. We assume that the crystal is oriented in such a way that $\boldsymbol{e}_{3}$ corresponds to the dislocation axis and $\boldsymbol{e}_{1}$ is collinear with the edge component of the Burgers vector $\left(b_{2}=0\right)$. With such an orientation, the tensor $\mathbf{K}^{0}$ appearing in the elastic energy of an isolated dislocation (Eq. 2) or of a dislocation dipole (Eq. 4) writes [20]

$$
\mathbf{K}^{0}=\frac{\mu}{2 \pi(1-\nu)}\left(\begin{array}{ccc}
1 & 0 & 0 \\
0 & 1 & 0 \\
0 & 0 & (1-\nu)
\end{array}\right),
$$

where $\mu$ is the shear modulus and $\nu$ Poisson's ratio.

The core traction contribution has been calculated by Bullough and Foreman [1]:

$$
E_{\mathrm{c}}^{\mathrm{elas}}(\phi)=\frac{\mu b_{1}^{2}}{16 \pi(1-\nu)}\left[\frac{1}{1-\nu}-2 \cos (2 \phi)\right]
$$

One sees that this contribution is null for a pure screw dislocation $\left(b_{1}=0\right)$, but this result does not hold anymore when elastic anisotropy is considered (§ (4). For a pure edge or a mixed dislocation, if the dislocation cut corresponds to the dislocation glide plane, one has then $\phi=0$ or $\phi=\pi$, and one recovers the expression given by Hirth and Lothe (Eq. (3-53) in Ref. [20]):

$$
E_{\mathrm{c}}^{\mathrm{elas}}(\phi=0)=-\frac{\mu b_{1}^{2}}{16 \pi} \frac{1-2 \nu}{(1-\nu)^{2}} \text {. }
$$

As Poisson's ratio is smaller than $1 / 2$, this shows that the core traction contribution reduces the dislocation elastic energy when the dislocation cut corresponds to its glide plane.

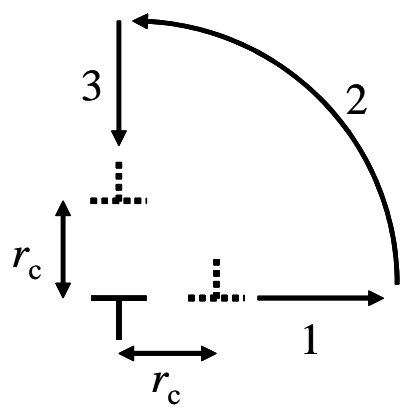

Figure 2. Reversible thermodynamic cycle for a dipole of edge dislocations. 
To understand the importance of the core traction contribution, one can look at the variation of energy for a dipole of edge dislocations subjected to the cycle sketched in Fig. 2. This cycle has been proposed by Hirth and Lothe (Ref. [2] and section 8-3 in Ref. [20]). The dipole is first created with both edge dislocations lying on the same glide plane and separated by a distance $r_{\mathrm{c}}$. The energy required to create the dipole is $2 E^{\text {core }}+2 E_{\mathrm{c}}^{\text {elas }}(\phi=0)$, where $E^{\text {core }}$ is the dislocation core energy, i.e. the part of energy which cannot be described by linear elasticity. One dislocation is then displaced through the cycle $1 \rightarrow 2 \rightarrow 3$. The work performed by the Peach-Koehler force during this displacement is $\mu b^{2}(1-\nu) / 2 \pi$. When the dislocations are at a distance $r_{\mathrm{c}}$, the dipole is destroyed and one recovers the energy $2 E^{\text {core }}+2 E_{\mathrm{c}}^{\text {elas }}(\phi=\pi / 2)$. The variation of energy through the complete cycle is then $2 E_{\mathrm{c}}^{\text {elas }}(\phi=\pi / 2)-2 E_{\mathrm{c}}^{\text {elas }}(\phi=0)+\mu b^{2}(1-\nu) / 2 \pi=0$. If one had neglected the core traction contribution to the dipole elastic energy, the energy variation would have been non-null which violates the law of thermodynamics. The proper consideration of all energy contributions in the Volterra elastic field thus allows a coherent definition of dislocation energetics. Such a coherency does not require more complex descriptions which assume a spreading of the dislocation like in the Peierls-Nabarro model or in the standard core model proposed by Lothe [21].

\section{Anisotropic elastic media}

\subsection{Sextic formalism}

We consider a dislocation of Burgers vector $\boldsymbol{b}$ located at the origin and we orient the crystal so that the axis $\boldsymbol{e}_{3}$ corresponds to the dislocation line. We assume that the angle $\phi$ in Fig. 1 is equal to $-\pi$ : the dislocation cut corresponds with the half plane defined by $x_{1}<0$ and $x_{2}=0$. We will generalize in a second stage to a different angle $\phi$.

The elastic displacement and the elastic stress created by the dislocation are given to the first order by the Volterra solution. They can be expressed using the formalism f] developed by Eshelby et al. [四] and extended by Stroh [5, 6]. The displacement and the stress calculated in a point of Cartesian coordinates $\left(x_{1}, x_{2}, x_{3}\right)$ are then

$$
\begin{aligned}
u_{k}\left(x_{1}, x_{2}\right) & =\frac{1}{2} \sum_{\alpha=1}^{6} \mp \frac{1}{2 \pi \mathrm{i}} A_{k}^{\alpha} D_{\alpha} \log \left(x_{1}+p_{\alpha} x_{2}\right) \\
\sigma_{i j}\left(x_{1}, x_{2}\right) & =\frac{1}{2} \sum_{\alpha=1}^{6} \mp \frac{1}{2 \pi \mathrm{i}} B_{i j k}^{\alpha} A_{k}^{\alpha} D_{\alpha} \frac{1}{x_{1}+p_{\alpha} x_{2}}
\end{aligned}
$$

The sign $\mp$ appearing in these equations means - for $1 \leq \alpha \leq 3$ and + for $4 \leq \alpha \leq 6$.

The matrices $B_{i j k}^{\alpha}$ are obtained from the elastic constants $C_{i j k l}$ expressed in the dislocation reference frame:

$$
B_{i j k}^{\alpha}=C_{i j k 1}+p_{\alpha} C_{i j k 2}
$$

\footnotetext{
${ }^{1}$ For a more detailled presentation of the sextic formalism, cf. chap. 13 in Ref, [2] of Refs. [1 and [22].

${ }^{2}$ We use the same sion convention as Hirth and Lothe 20]. Eshelby et al. 4 and Stroh [5, 6 , use the opposite sign in Eq. 6 .
} 
The roots $p_{\alpha}$ are solution of the sextic equations corresponding to the following determinant being equal to zero

$$
\left|\left\{B_{i 1 k}^{\alpha}+p_{\alpha} B_{i 2 k}^{\alpha}\right\}\right|=0
$$

and the non-null vectors $A_{k}^{\alpha}$, associated to each root $p_{\alpha}$, check the following equation

$$
\left(B_{i 1 k}^{\alpha}+p_{\alpha} B_{i 2 k}^{\alpha}\right) A_{k}^{\alpha}=0 .
$$

In all equations, we use the Einstein summation convention on repeated indexes, except for indexes written in Greek letters. When summation on the six different roots $p_{\alpha}$ is required, it will be explicitly written like in Eq. 6.

The six roots $p_{\alpha}$ are necessary complex. If $p_{\alpha}$ is solution of Eq. 7, its complex conjugate $p_{\alpha}{ }^{*}$ is also a solution of this equation. We can therefore assume that the six different roots have been sorted in such a way that

$$
\Im\left(p_{\alpha}\right)>0 \text { and } p_{\alpha+3}=p_{\alpha}{ }^{*}, \quad 1 \leq \alpha \leq 3,
$$

where $\Im\left(p_{\alpha}\right)$ is the imaginary part of $p_{\alpha}$.

The elastic field given by Eq. 6 corresponds to the one of a dislocation with a Burgers vector $\boldsymbol{b}$ and a line direction $\boldsymbol{e}_{3}$ if the constants $D_{\alpha}$ check the equations:

$$
\begin{aligned}
\frac{1}{2} \sum_{\alpha=1}^{6} A_{k}^{\alpha} D_{\alpha} & =-b_{k}, \\
\frac{1}{2} \sum_{\alpha=1}^{6} B_{i 2 k}^{\alpha} A_{k}^{\alpha} D_{\alpha} & =0 .
\end{aligned}
$$

We choose the principal determination for the complex logarithm appearing in Eq. 6. The elastic displacement created by the dislocation therefore presents a discontinuity in the half plane of equations $x_{1}<0$ and $x_{2}=0$ defining the dislocation cut.

The system of linear equations 9 can be easily solved following Stroh method [5, 6]. To do so, we define a new vector

$$
L_{i}^{\alpha}=B_{i 2 k}^{\alpha} A_{k}^{\alpha}=-\frac{1}{p_{\alpha}} B_{i 1 k}^{\alpha} A_{k}^{\alpha} .
$$

Both definitions are equivalent because of Eq. 1. As the vector $A_{k}^{\alpha}$ is an eigenvector defined by Eq. 0, its norm is not fixed. One can therefore choose it so as to check the following normalization condition:

$$
2 A_{i}^{\alpha} L_{i}^{\alpha}=1, \quad \forall \alpha
$$

Using the orthogonality properties [5, 6, 0, 22] of the vectors $A_{i}^{\alpha}$ and $L_{i}^{\alpha}$, the solution of the system of equations 9 is given by

$$
D_{\alpha}=-2 L_{i}^{\alpha} b_{i}
$$

With such definitions, the tensor $\mathbf{K}^{0}$ appearing in the elastic energy of an isolated 
dislocation (Eq. 2) or of a dislocation dipole (Eq. 14) is given by

$$
K_{i j}^{0}=\sum_{\alpha=1}^{6} \pm \frac{1}{2 \pi \mathrm{i}} L_{i}^{\alpha} L_{j}^{\alpha} .
$$

\subsection{The core tractions contribution}

The contribution of the core tractions to the elastic energy (Eq. 3i) is given by

$$
E_{\mathrm{c}}^{\text {elas }}=-\frac{1}{2} \int_{\theta=-\pi}^{\pi}\left[\sigma_{i 1}\left(r_{\mathrm{c}}, \theta\right) \cos (\theta)+\sigma_{i 2}\left(r_{\mathrm{c}}, \theta\right) \sin (\theta)\right] u_{i}\left(r_{\mathrm{c}}, \theta\right) r_{\mathrm{c}} \mathrm{d} \theta .
$$

The expression 6 shows that the elastic displacement $\boldsymbol{u}(r, \theta)$ is the sum of an angular function and a term depending only on $\ln \left(r_{\mathrm{c}}\right)$. This last term leads to a contribution in Eq. 13 which is null because the resultant of forces located in the core is null for a pure dislocation [5, 6]. Only the angular part of the elastic displacement leads to a contribution in Eq. 13. One thus obtains

$$
\begin{aligned}
E_{\mathrm{c}}^{\mathrm{elas}}=-\frac{1}{8} \sum_{\alpha=1}^{6} \mp \frac{1}{2 \pi \mathrm{i}} A_{i}^{\alpha} D_{\alpha} \sum_{\beta=1}^{6} \mp \frac{1}{2 \pi \mathrm{i}} A_{k}^{\beta} D_{\beta} \\
\int_{-\pi}^{\pi} \frac{B_{i 1 k}^{\beta} \cos (\theta)+B_{i 2 k}^{\beta} \sin (\theta)}{\cos (\theta)+p_{\beta} \sin (\theta)} \log \left[\cos (\theta)+p_{\alpha} \sin (\theta)\right] \mathrm{d} \theta .
\end{aligned}
$$

This expression already shows that the contribution of the core tractions to the elastic energy does not depend on the radius $r_{\mathrm{c}}$ of the core cylinder. Using the property 7 of the matrices $B_{i j k}^{\beta}$ and the vectors $A_{k}^{\beta}$, as well as the definition 10 of the vector $L_{i}^{\beta}$, one gets

$$
E_{\mathrm{c}}^{\mathrm{elas}}=\frac{1}{8} \sum_{\alpha=1}^{6} \pm \frac{1}{2 \pi \mathrm{i}} \sum_{\beta=1}^{6} \pm \frac{1}{2 \pi \mathrm{i}} D_{\alpha} A_{i}^{\alpha} L_{i}^{\beta} D_{\beta} J^{1}\left(p_{\alpha}, p_{\beta}\right),
$$

where the integral $J^{1}(p, a)$ is defined by

$$
J^{1}(p, q)=\int_{-\pi}^{\pi} \frac{-p \cos \theta+\sin \theta}{\cos \theta+p \sin \theta} \log (\cos \theta+q \sin \theta) \mathrm{d} \theta .
$$

An analytical expression of this integral is obtained in the appendix A. Using this expression with the properties 8 checked by the roots $p_{\alpha}$, one obtains

$$
\begin{aligned}
E_{\mathrm{c}}^{\mathrm{elas}}=\frac{1}{8} \sum_{\alpha=1}^{6} \log \left(\mathrm{i} \pm p_{\alpha}\right) \sum_{\beta=1}^{6} \pm \frac{1}{2 \pi \mathrm{i}} D_{\alpha}\left(A_{i}^{\alpha} L_{i}^{\beta}-L_{i}^{\alpha} A_{i}^{\beta}\right) D_{\beta} \\
+\frac{1}{8 \pi \mathrm{i}} \sum_{\alpha=1}^{3} \sum_{\beta=4}^{6} D_{\alpha}\left(A_{i}^{\alpha} L_{i}^{\beta}-L_{i}^{\alpha} A_{i}^{\beta}\right) D_{\beta} \log \left(p_{\alpha}-p_{\beta}\right) .
\end{aligned}
$$

We therefore obtained an analytical expression of the core traction contribution within the sextic formalism. 


\subsection{Angular dependence of the dipole elastic energy}

We now examine how the dipole elastic energy varies when it is rotated with respect to the crystallographic axes. The answer could be obtained by rotating the dislocations and the elastic constants so as to calculate in the new reference frame all quantities needed to express the elastic energy. But a closed-form expression of the angular dependence of the elastic energy can be obtained in a fixed reference frame. We will show that this expression is consistent with the dislocation interaction energy derived by Stroh [0].

The angle $\phi$ can now deviate from the value $-\pi$ considered above. A rotation of the dislocation dipole is equivalent to a rotation of the corresponding cut (Fig. 1). Such a rotation does not modify the vectors $A_{i}^{\alpha}$ and $L_{i}^{\alpha}[$ [7, 22]. As a consequence, the tensor $\mathbf{K}^{0}$ appearing in the dipole elastic energy (Eq. đ) is unchanged. Only the contribution $E_{\mathrm{c}}^{\text {elas }}$ of the dislocation core tractions depends on this angle $\phi$. We then call $\Delta E_{\mathrm{c}}^{\text {elas }}(\phi)$ the variation of the dipole elastic energy, with the convention $\Delta E_{\mathrm{c}}^{\mathrm{elas}}(-\pi)=0$. To calculate this energy variation, it is useful to write the roots of the sextic equations in the form $p_{\alpha}=\tan \left(\psi_{\alpha}\right)$. Such a transformation can be performed as long as the roots differ from $\pm \mathrm{i}$ which only happens in degenerate cases due to some isotropy of the elastic constants] [7]. We can now rewrite the integral (Eq. 15) appearing in the energy contribution of the core tractions (Eq. 14)

$$
J^{1}\left(p_{\alpha}, p_{\beta}\right)=\int_{-\pi}^{\pi}-\tan \left(\psi_{\alpha}-\theta\right) \log \left[\frac{\cos \left(\psi_{\beta}-\theta\right)}{\cos \left(\psi_{\beta}\right)}\right] \mathrm{d} \theta .
$$

The rotation of the cut modify the roots through the relation $p_{\alpha}(\phi)=\tan \left(\psi_{\alpha}-\phi\right)$ [7]. As a consequence, the elastic energy variation is given by

$$
\Delta E_{\mathrm{c}}^{\mathrm{elas}}(\phi)=\frac{1}{8} \sum_{\alpha=1}^{6} \pm \frac{1}{2 \pi \mathrm{i}} \sum_{\beta=1}^{6} \pm \frac{1}{2 \pi \mathrm{i}} D_{\alpha} A_{i}^{\alpha} L_{i}^{\beta} D_{\beta} \Delta J_{\phi}^{1}\left(p_{\alpha}, p_{\beta}\right),
$$

with

$$
\begin{aligned}
\Delta J_{\phi}^{1}\left(p_{\alpha}, p_{\beta}\right)= & \int_{-\pi}^{\pi}-\tan \left(\psi_{\alpha}-\theta\right)\left\{\log \left[\frac{\cos \left(\psi_{\beta}-\theta\right)}{\cos \left(\psi_{\beta}-\phi\right)}\right]-\log \left[\frac{\cos \left(\psi_{\beta}-\theta\right)}{\cos \left(\psi_{\beta}\right)}\right]\right\} \mathrm{d} \theta \\
= & \int_{-\pi}^{\pi} \frac{-p_{\alpha} \cos \theta+\sin \theta}{\cos \theta+p_{\alpha} \sin \theta} \\
& {\left[\log \left(\frac{\cos \theta+p_{\beta} \sin \theta}{\cos \phi+p_{\beta} \sin \phi}\right)-\log \left(\cos \theta+p_{\beta} \sin \theta\right)\right] \mathrm{d} \theta }
\end{aligned}
$$

This integral is calculated in the appendix B. With the result of this appendix, one obtains

$$
\begin{aligned}
\Delta E_{\mathrm{c}}^{\mathrm{elas}}(\phi)= & \frac{1}{8} \sum_{\alpha=1}^{6} \sum_{\beta=1}^{6} \pm \frac{1}{2 \pi \mathrm{i}} D_{\alpha} A_{i}^{\alpha} L_{i}^{\beta} D_{\beta} \log \left(\cos \phi+p_{\beta} \sin \phi\right) \\
& -\frac{1}{8} \sum_{\alpha=1}^{6} \pm \frac{1}{2 \pi \mathrm{i}} \sum_{\beta=1}^{6} D_{\alpha} A_{i}^{\alpha} L_{i}^{\beta} D_{\beta} \log \left(\cos \phi+p_{\alpha} \sin \phi\right) .
\end{aligned}
$$

\footnotetext{
${ }^{1}$ The degeneracy can be lifted by adding some noise to the elastic constants.
} 
Using the orthogonality properties checked by the vectors $A_{i}^{\alpha}$ and $L_{i}^{\alpha}$, as well as the definition 11 of $D_{\alpha}$, one gets

$$
\Delta E_{\mathrm{c}}^{\mathrm{elas}}(\phi)=\frac{1}{2} \sum_{\alpha=1}^{6} \pm \frac{1}{2 \pi \mathrm{i}} b_{i} L_{i}^{\alpha} L_{j}^{\alpha} b_{j} \log \left(\cos \phi+p_{\alpha} \sin \phi\right) .
$$

One recovers an angular dependence which is in agreement with the expression of the interaction energy between two dislocations given by Stroh [5], as well as by Cai et al. $[8]$. I

This expression 17 shows too that the core traction contribution $E_{\mathrm{c}}^{\text {elas }}$ is periodic of period $\pi$, i.e. is insensitive to an inversion of the cut direction. We have indeed

$$
\Delta E_{\mathrm{c}}^{\mathrm{elas}}(\phi+\pi)=\frac{1}{2} \sum_{\alpha=1}^{6} \pm \frac{1}{2 \pi \mathrm{i}} b_{i} L_{i}^{\alpha} L_{j}^{\alpha} b_{j} \log \left(-\cos \phi-p_{\alpha} \sin \phi\right) .
$$

Using the property of $\log (-z)$, we obtain

$$
\begin{aligned}
\Delta E_{\mathrm{c}}^{\text {elas }}(\phi+\pi) & =\Delta E_{\mathrm{c}}^{\text {elas }}(\phi)-\frac{1}{4} \sum_{\alpha=1}^{6} b_{i} L_{i}^{\alpha} L_{j}^{\alpha} b_{j} & & \text { if } 0<\phi<\pi \\
& =\Delta E_{\mathrm{c}}^{\text {elas }}(\phi)+\frac{1}{4} \sum_{\alpha=1}^{6} b_{i} L_{i}^{\alpha} L_{j}^{\alpha} b_{j} & & \text { if }-\pi<\phi<0 .
\end{aligned}
$$

Finally, the closure properties of the vectors $L_{i}^{\alpha}[6,6,22]$ lead to the desired result:

$$
\Delta E_{\mathrm{c}}^{\mathrm{elas}}(\phi+\pi)=\Delta E_{\mathrm{c}}^{\mathrm{elas}}(\phi) \text {. }
$$

\section{Edge dislocations in $\alpha$-iron}

So as to illustrate the need to take into account the contribution of core tractions to the dislocation elastic energy, we study now edge dislocations in $\alpha$-iron, and determine their core energies $E^{\text {core }}$. The core energy is the amount of the dislocation excess energy that arises from atomic interaction close to the dislocation core that cannot be described by linear elasticity. This is therefore a supplementary energy contribution that should be added to the elastic energy. One expects that such an energy contribution is an intrinsic property of the dislocation: its value should only depend on the dislocation and not on the surrounding environment, like other dislocations.

We use the Fe empirical potential developed by Mendelev et al. [23] in its modified version published in Ref. [24]. Thanks to the existence of a cut-off for the interactions between atoms, two different methods can be used to determine the dislocation core energy. One can either work with an isolated dislocation in an infinite elastic medium (the cluster approach), or with a dislocation dipole in periodic boundary conditions (the dipole approach). In the following section, we will show

\footnotetext{
${ }^{1}$ The expression used by $\mathrm{Li}$ et al. [9], based on the work of Cai et al. [8, is different from Eq. 17. Li obtained $\Delta E^{c}(\phi)=\frac{1}{2 \pi} \sum_{\alpha=1}^{3} \Im\left\{b_{i} L_{i}^{\alpha} L_{j}^{\alpha} b_{j}\right\} \Re\left\{\log \left(\cos \phi+p_{\alpha} \sin \phi\right)\right\}$, whereas Eq. 17 can be rewritten $\Delta E^{c}(\phi)=\frac{1}{2 \pi} \sum_{\alpha=1}^{3} \Im\left\{b_{i} L_{i}^{\alpha} L_{j}^{\alpha} b_{j} \log \left(\cos \phi+p_{\alpha} \sin \phi\right)\right\}$. Both definitions may be equivalent in the case of a screw dislocation studied by Li et al., but we could not demonstrate it.
} 
that both methods are coherent and lead to the same core energy as long as all contributions are considered in the elastic energy.

$\alpha$-Fe has a body-centered cubic lattice and the Burgers vector of the most common dislocations is $\boldsymbol{b}=a / 2\langle 111\rangle$, where $a=2.8553 \AA$ is the lattice vector. Depending on the temperature, different slip planes are observed, either $\{110\}$ or $\{112\}$ planes. It is therefore interesting to study the energetics of both $1 / 2\langle 111\rangle\{110\}$ and $1 / 2\langle 111\rangle\{112\}$ edge dislocations, which both can exist. We will therefore determine the core energy of these two dislocations. For the $1 / 2\langle 111\rangle\{110\}$ edge dislocation, we use a simulation box with the orientation $\boldsymbol{e}_{1}=[111] / \sqrt{3}, \boldsymbol{e}_{2}=[\overline{101}] / \sqrt{2}$, and $\boldsymbol{e}_{3}=[1 \overline{2} 1] / \sqrt{6}$; for the $1 / 2\langle 111\rangle\{112\}$ edge dislocation $\boldsymbol{e}_{1}=[111] / \sqrt{3}$, $\boldsymbol{e}_{2}=[\overline{1} 2 \overline{1}] / \sqrt{6}$, and $\boldsymbol{e}_{3}=[\overline{1} 01] / \sqrt{2}$. In both cases, $\boldsymbol{e}_{1}$ is collinear with the Burgers vector and $\boldsymbol{e}_{3}$ corresponds to the dislocation line. The dislocation glide plane is therefore the plane of normal $\boldsymbol{e}_{2}(\phi=0$ in Fig. 1).

\subsection{The cluster approach}

In the cluster approach, a single dislocation is introduced at the center of a unit cell which is periodic only along the dislocation line and with surface in other directions. Atoms are displaced according to the anisotropic elastic Volterra displacement (Eq. 6). Atoms closer from the external surface than the interatomic potential cut-off are kept fixed while the positions of inner atoms are relaxed so as to minimize the energy calculated with the potential. This therefore simulates an isolated dislocation in an infinite elastic medium. A variant of the method consists in relaxing atoms at the surface using lattice Green functions [25, 26, 27]. This may be necessary in ab-initio calculations because of the small size of the unit cell that can be simulated. As, in the present work, we use empirical potential, the unit cell is large enough so that the Volterra elastic field correctly describes the displacements of atoms at the surface and one does not need to use lattice Green function to relax them.

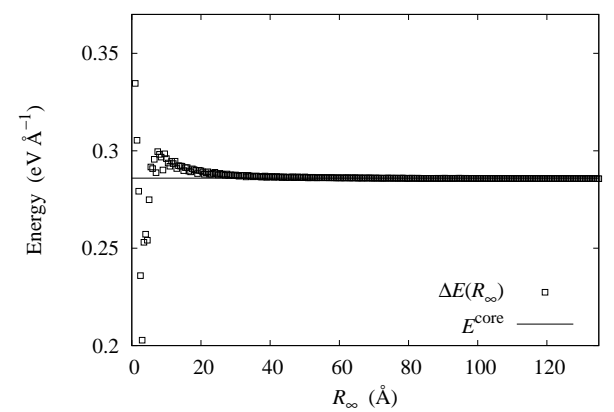

(a) $1 / 2\langle 111\rangle\{110\}$

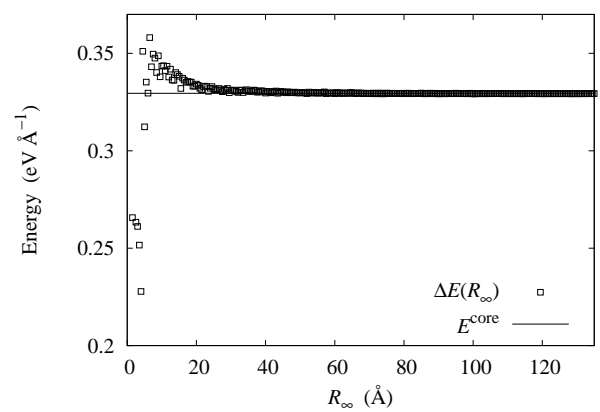

(b) $1 / 2\langle 111\rangle\{112\}$

Figure 3. Variation with the cylinder radius $R_{\infty}$ of the energy difference $\Delta E\left(R_{\infty}\right)=E\left(R_{\infty}\right)-$ $\frac{1}{2} b_{i} K_{i j}^{0} b_{j} \ln \left(R_{\infty} / r_{\mathrm{c}}\right)$ showing the convergence to $E^{\text {core }}$ for $1 / 2\langle 111\rangle\{110\}$ and $1 / 2\langle 111\rangle\{112\}$ edge dislocations in $\alpha$-iron $\left(r_{\mathrm{c}}=b \simeq 2.473 \AA\right.$ 趹.

Once inner atoms are relaxed, we calculate the excess energy $E\left(R_{\infty}\right)$ given by the interatomic potential and contained in a cylinder centered on the dislocation, of axis $\boldsymbol{e}_{3}$ and of radius $R_{\infty}$. This excess energy is the difference of energy between the system with the dislocation and the perfect crystal for the same number of atoms. Only the excess energy of atoms contained in the cylinder is considered. This can be performed for different values of the radius $R_{\infty}$ in the limit of the 
Table 1. Parameters defining the energies of both edge dislocations in $\alpha$-iron. All quantities are given in $\mathrm{meV} \mathrm{A}^{-1}$. The core energies are given for a core radius $r_{\mathrm{c}}=b$.

\begin{tabular}{lccc}
\hline & $E^{\text {core }}$ & $E_{\mathrm{c}}^{\text {elas }}(\phi=0)$ & $\frac{1}{2} b_{i} K_{i j}^{0} b_{j}$ \\
\hline $1 / 2\langle 111\rangle\{110\}$ & 286 & -87 & 370 \\
$1 / 2\langle 111\rangle\{112\}$ & 329.5 & -89 & 378 \\
\hline
\end{tabular}

maximal radius allowed by the unit cell. According to the section 2 (Eq. 2), this excess energy should vary as

$$
E\left(R_{\infty}\right)=E^{\mathrm{core}}+\frac{1}{2} b_{i} K_{i j}^{0} b_{j} \ln \left(\frac{R_{\infty}}{r_{\mathrm{c}}}\right) .
$$

In this expression, the tensor $\mathbf{K}^{0}$ is given by Eq. 12. It is different for the $1 / 2\langle 111\rangle\{110\}$ and $1 / 2\langle 111\rangle\{112\}$ edge dislocations because of elastic anisotropy (Tab. 1). Looking at the variations of the energy difference $\Delta E\left(R_{\infty}\right)=E\left(R_{\infty}\right)-$ $\frac{1}{2} b_{i} K_{i j}^{0} b_{j} \ln \left(R_{\infty} / r_{\mathrm{c}}\right)$, one expects this quantity to be a constant defining $E^{\mathrm{core}}$. This quantity actually varies but rapidly converges to a constant value for an increasing radius $R_{\infty}$ (Fig. 3). Variations for small $R_{\infty}$ arises from the dislocation core field, which may be important close to the dislocation core but is not taken into account in the present approach. This core field needs only to be considered when one cannot reach large enough simulation boxes like in ab initio calculations [18]. The convergence of $\Delta E\left(R_{\infty}\right)$ allows us anyway to define a dislocation core energy. Values of this core energy are given for both dislocation in Tab. 1.

\subsection{The dipole approach}

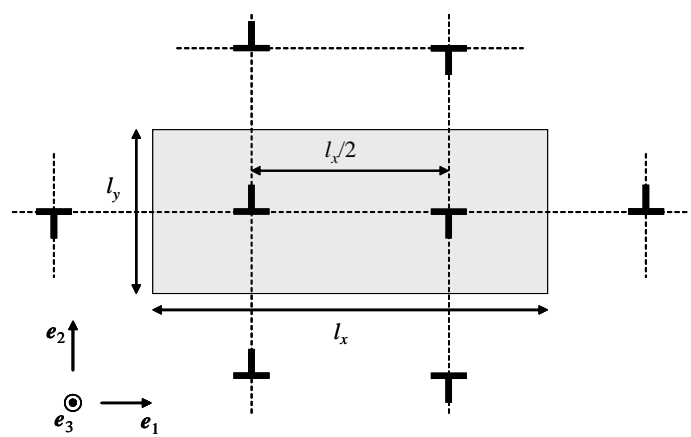

Figure 4. Sketch of the periodic unit cell used to study a dipole of edge dislocations.

In the dipole approach, full periodic boundary conditions are used. The total Burgers vector of the unit cell has to be zero. Therefore a dislocation dipole is introduced in the unit cell. The periodic unit cell we used for the present work is sketched in Fig. A. Both dislocations composing the dipole share the same glide plane and then $\phi=0$.

The energy of the unit cell is minimized by relaxing all atomic positions. According to section 2, the excess energy of the unit cell should be

$$
E=2 E^{\text {core }}+2 E_{\mathrm{c}}^{\mathrm{elas}}(\phi=0)+b_{i} K_{i j}^{0} b_{j} \ln \left(\frac{d}{r_{\mathrm{c}}}\right)+E_{\text {inter }}^{\text {elas }},
$$

with $d=l_{x} / 2 . E_{\text {inter }}^{\text {elas }}$ is the elastic interaction energy between the dislocation dipole 


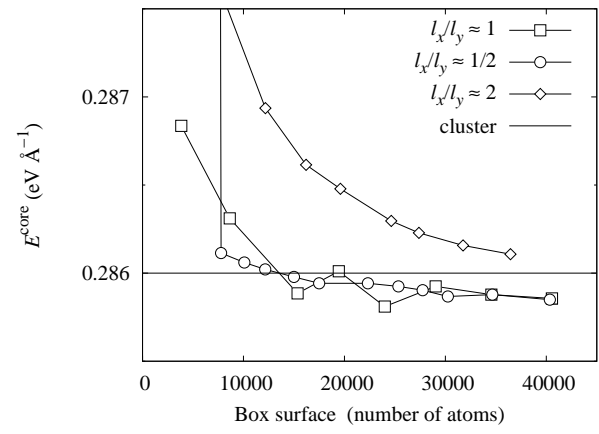

(a) $1 / 2\langle 111\rangle\{110\}$

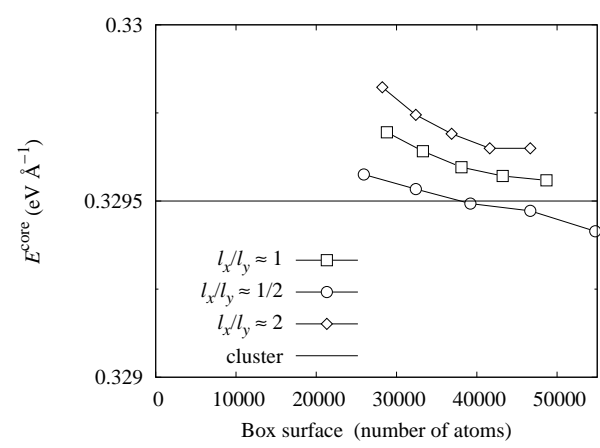

(b) $1 / 2\langle 111\rangle\{112\}$

Figure 5. Variation with the size of the simulation cell of the dislocation core energy deduced from atomic simulations of a dislocation dipole in periodic boundary conditions for $1 / 2\langle 111\rangle\{110\}$ and $1 / 2\langle 111\rangle\{112\}$ edge dislocations in $\alpha$-iron $\left(r_{\mathrm{c}}=b \simeq 2.473 \AA \mathrm{A}\right)$. Symbols correspond to result of the dipole approach for different geometries of the unit cell and the horizontal line to the result of the cluster approach.

contained in the unit cell and all its periodic images. This elastic interaction energy is calculated using the method of Cai et al. [8]. One can therefore deduce the core energy from the atomic simulations using Eq. 18: the excess energy $E$ is directly given by atomic simulations and all elastic contributions are calculated using a code based on anisotropic linear elasticity.

The obtained core energies are presented in figure 5 for different sizes of the unit cells as well as different geometries characterized by the aspect ratio $l_{x} / l_{y}$. All geometries converge with the size of the unit cell to the same limit and the converged value of the core energy is in perfect agreement with the value obtained in the cluster approach. The table 1 shows that this agreement is possible only if one does not forget the contribution of the core tractions to the elastic energy. Without this contribution, the core energies would have been about $90 \mathrm{meV} \mathrm{A}^{-1}$ lower in the dipole approach than in the cluster approach.

\subsection{Stability of an edge dipole}

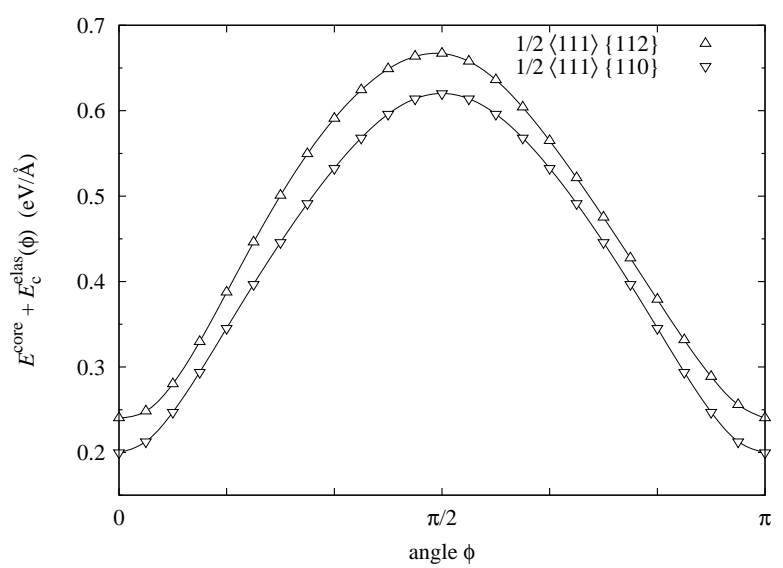

Figure 6. Variation with the angle $\phi$ of the energy contribution $E^{\text {core }}+E_{\mathrm{c}}^{\text {elas }}(\phi)\left(r_{\mathrm{c}}=b\right)$.

Eq. 18 shows that the energy of a dislocation dipole is the sum of a constant term $\left(E^{\text {core }}+E_{\mathrm{c}}^{\text {elas }}(\phi)\right)$ and a term depending on the distance $d$ between the two dislocations composing the dipole. The values obtained from atomic simulations 
and anisotropic linear elasticity (Tab. 1) shows that the $1 / 2\langle 111\rangle\{110\}$ edge dipole is more stable than the $1 / 2\langle 111\rangle\{112\}$ one, whatever the distance $d$. This result is strictly valid only when both dislocations share the same glide plane $(\phi=0)$. We now need to look to the variation of the distant independent term $E^{\text {core }}+E_{\mathrm{c}}^{\text {elas }}(\phi)$ with the angle $\phi$, i.e. with the orientation of the dipole.

Fig. 6 shows the variation of $E^{\text {core }}+E_{\mathrm{c}}^{\text {elas }}(\phi)$ with the angle $\phi$ for both dislocation. Whatever the orientation of the dipole, this energy contribution is smaller for the $1 / 2\langle 111\rangle\{110\}$ than for the $1 / 2\langle 111\rangle\{112\}$ edge dipole. The $1 / 2\langle 111\rangle\{110\}$ is therefore found as the most stable edge dipole.

\section{Conclusions and discussion}

We obtained within the sextic formalism an expression of the core traction contribution to the elastic energy (Eq. 16). We showed that this expression agrees with the angular dependence of the interaction energy between two dislocations previously derived by Stroh [5]. This energy contribution is actually important so that the dislocation elastic energy can be a state variable consistent with the work of the Peach-Koehler forces.

This contribution to the elastic energy needs also to be considered when one wants to extract from atomic simulations core energies which does not depend on the simulation conditions. Both the core traction contribution and the core energy do not depend on the length scale of the dislocation microstructure. Their physical meaning is nevertheless different. The core energy is a dislocation intrinsic property which takes into account the fact that atomic interactions cannot be described by elasticity close to the dislocation core. On the other hand, the core traction contribution is a part of the elastic energy and depends on the positions of other dislocations through the angle $\phi$ defining the dislocation cut.

The application to edge dislocations in iron showed that dislocation core energies could be obtained consistently from different simulation approaches, i.e. the cluster or the dipole approaches. Both approaches lead to the same core energy when one does not forget to take into account the energy contribution of core tractions in the dipole approach. This allowed us to conclude that $1 / 2\langle 111\rangle\{110\}$ edge dislocations are more stable than $1 / 2\langle 111\rangle\{112\}$ ones.

The contribution of core tractions is also important when defining the elastic energy of a dislocation loop as pointed by Bullough and Foreman [1]. For a glissile loop, this contribution generally reduces the elastic energy of edge segments: this is true at least for an isotropic crystal (Eq. 5). As a consequence, this would lead to loop shapes which are more rounded than with a simple line tension model where this contribution is omitted [28]. As this contribution does not depend on the loop size, smaller loops should be more rounded than larger ones, in agreement with experimental observations [29]. This energy contribution could therefore explain some discrepancies obtained between a simple line tension model and, either experimental observations [29], or results deduced from simulations of the loop self stress [30].

Gavazza and Barnett [3] showed that part of the self-force acting on a loop is associated with the core traction contribution to the elastic energy. The obtained expression of this contribution to the elastic energy (Eq. 16) could therefore be used in dislocation dynamics simulations to compute the self-force acting on a dislocation segment.

To conclude, we would like to stress that we obtained the expression of the core traction contribution to the elastic energy within the framework of the sextic formalism. Anisotropic linear elasticity of line defects can also be handled within 
the integral formalism[7, 22]. In the integral method, all quantities defining the elastic fields are obtained from angular integrals, so that one does not need to look for the solutions of the sextic equations. The recasting of our result within the integral formalism stills needs to be done. But this may not be possible as the expression 16 we obtained does not make appear any known integral. Getting an expression of the core traction contribution to the elastic energy within the integral formalism may be therefore a challenge.

\section{Acknowledgments}

The author is grateful to V. Bulatov, L. Ventelon, and F. Willaime for stimulating discussions, as well as to B. Lemoine for his help with the atomic simulations.

\section{Appendix A. Integral $J^{1}(p, q)$}

In this appendix, we calculate the integral appearing in the contribution to the dislocation elastic energy of the core tractions:

$$
J^{1}(p, q)=\int_{-\pi}^{\pi} \frac{-p \cos \theta+\sin \theta}{\cos \theta+p \sin \theta} \log (\cos \theta+q \sin \theta) \mathrm{d} \theta .
$$

An integration by parts leads to

$$
\begin{aligned}
J^{1}(p, q)=-[\log (\cos \theta+p \sin \theta) & \log (\cos \theta+q \sin \theta)]_{-\pi}^{\pi} \\
& +\int_{-\pi}^{\pi} \log (\cos \theta+p \sin \theta) \frac{-\sin \theta+q \cos \theta}{\cos \theta+q \sin \theta} \mathrm{d} \theta .
\end{aligned}
$$

We can conclude that

$$
J^{1}(p, q)=-J^{1}(q, p)
$$

It is not possible to directly perform the integration, because of the logarithm function appearing in the definition of $J^{1}(p, q)$. To circumvent the problem, we derive $J^{1}(p, q)$ with respect to the parameter $q$

$$
\frac{\partial J^{1}(p, q)}{\partial q}=\int_{-\pi}^{\pi} \frac{-p \cos \theta+\sin \theta}{\cos \theta+p \sin \theta} \frac{\sin \theta}{\cos \theta+q \sin \theta} \mathrm{d} \theta .
$$

We thus obtain an integral of a rational function of $\cos (\theta)$ and $\sin (\theta)$. It can be integrated using the residues theorem [31]:

$$
\begin{aligned}
\frac{\partial J^{1}(p, q)}{\partial q} & =-\frac{2 \pi \mathrm{i}}{\mathrm{i}+q} & & \text { if } \Im(p)>0 \text { and } \Im(q)>0, \\
& =-\frac{2 \pi \mathrm{i}}{\mathrm{i}-q}+\frac{4 \pi \mathrm{i}}{p-q} & & \text { if } \Im(p)>0 \text { and } \Im(q)<0, \\
& =-\frac{2 \pi \mathrm{i}}{\mathrm{i}+q}+\frac{4 \pi \mathrm{i}}{q-p} & & \text { if } \Im(p)<0 \text { and } \Im(q)>0, \\
& =-\frac{2 \pi \mathrm{i}}{\mathrm{i}-q} & & \text { if } \Im(p)<0 \text { and } \Im(q)<0 .
\end{aligned}
$$


Now, we can integrate this result with respect to the parameter $q$ :

$$
\begin{aligned}
J^{1}(p, q) & =-2 \pi \mathrm{i} \log (\mathrm{i}+q)+f_{1}(p) & & \text { if } \Im(p)>0 \text { and } \Im(q)>0, \\
& =2 \pi \mathrm{i} \log (\mathrm{i}-q)-4 \pi \mathrm{i} \log (p-q)+f_{2}(p) & & \text { if } \Im(p)>0 \text { and } \Im(q)<0, \\
& =-2 \pi \mathrm{i} \log (\mathrm{i}+q)+4 \pi \mathrm{i} \log (q-p)+f_{3}(p) & & \text { if } \Im(p)<0 \text { and } \Im(q)>0, \\
& =2 \pi \mathrm{i} \log (\mathrm{i}-q)+f_{4}(p) & & \text { if } \Im(p)<0 \text { and } \Im(q)<0,
\end{aligned}
$$

where $f_{1}, f_{2}, f_{3}$ and $f_{4}$ are four functions depending solely on the parameter $p$. They can be determined by using the property A1 and the result $J^{1}(\mathrm{i},-\mathrm{i})=2 \pi^{2}$. This leads to the final result:

$$
\begin{aligned}
J^{1}(p, q)= & 2 \pi \mathrm{i} \log (\mathrm{i}+p)-2 \pi \mathrm{i} \log (\mathrm{i}+q) & & \text { if } \Im(p)>0 \text { and } \Im(q)>0, \\
= & 2 \pi \mathrm{i} \log (\mathrm{i}+p)+2 \pi \mathrm{i} \log (\mathrm{i}-q) & & \\
& -4 \pi \mathrm{i} \log (p-q) & & \text { if } \Im(p)>0 \text { and } \Im(q)<0, \\
= & -2 \pi \mathrm{i} \log (\mathrm{i}-p)-2 \pi \mathrm{i} \log (\mathrm{i}+q) & & \\
& +4 \pi \mathrm{i} \log (q-p) & & \text { if } \Im(p)<0 \text { and } \Im(q)>0, \\
= & -2 \pi \mathrm{i} \log (\mathrm{i}-p)+2 \pi \mathrm{i} \log (\mathrm{i}-q) & & \text { if } \Im(p)<0 \text { and } \Im(q)<0 .
\end{aligned}
$$

\section{Appendix B. Integral $\Delta J_{\phi}^{1}(p, q)$}

The angular dependence of the dipole elastic energy makes appear the following integral

$$
\begin{aligned}
\Delta J_{\phi}^{1}(p, q)=\int_{-\pi}^{\pi} \frac{-p \cos \theta+\sin \theta}{\cos \theta+p \sin \theta} & \\
& {\left[\log \left(\frac{\cos \theta+q \sin \theta}{\cos \phi+q \sin \phi}\right)-\log (\cos \theta+q \sin \theta)\right] \mathrm{d} \theta . }
\end{aligned}
$$

We use the same integration method as for $J^{1}(p, q)$. A derivation with respect to the parameter $q$ leads to

$$
\frac{\partial}{\partial q} \Delta J_{\phi}^{1}(p, q)=\int_{-\pi}^{\pi} \frac{-p \cos \theta+\sin \theta}{\cos \theta+p \sin \theta} \frac{-\sin \phi}{\cos \phi+q \sin \phi} \mathrm{d} \theta .
$$

Using the residues theorem, we obtain

$$
\begin{aligned}
\frac{\partial}{\partial q} \Delta J_{\phi}^{1}(p, q) & =2 \pi \mathrm{i} \frac{\sin \phi}{\cos \phi+q \sin \phi} & & \text { if } \Im(p)>0, \\
& =-2 \pi \mathrm{i} \frac{\sin \phi}{\cos \phi+q \sin \phi} & & \text { if } \Im(p)<0 .
\end{aligned}
$$

We then integrate with respect to the parameter $q$ and we use the property $\Delta J_{\phi}^{1}(p, q)=-\Delta J_{\phi}^{1}(q, p)$ deduced from an integration by parts of the initial in- 
tegral. This leads to the final result

$$
\begin{aligned}
\Delta J_{\phi}^{1}(p, q)= & 2 \pi \mathrm{i} \log [\cos \phi+q \sin \phi] \\
& -2 \pi \mathrm{i} \log [\cos \phi+p \sin \phi] \quad \text { if } \Im(p)>0 \text { and } \Im(q)>0, \\
= & 2 \pi \mathrm{i} \log [\cos \phi+q \sin \phi] \\
& +2 \pi \mathrm{i} \log [\cos \phi+p \sin \phi] \quad \text { if } \Im(p)>0 \text { and } \Im(q)<0, \\
= & -2 \pi \mathrm{i} \log [\cos \phi+q \sin \phi] \\
& -2 \pi \mathrm{i} \log [\cos \phi+p \sin \phi] \quad \text { if } \Im(p)<0 \text { and } \Im(q)>0, \\
= & -2 \pi \mathrm{i} \log [\cos \phi+q \sin \phi] \\
& +2 \pi \mathrm{i} \log [\cos \phi+p \sin \phi] \quad \text { if } \Im(p)<0 \text { and } \Im(q)<0 .
\end{aligned}
$$

\section{References}

[1] R. Bullough and E.A.J. Foreman, Philos. Mag. 9 (1964) p.315-329.

[2] J. Lothe and J.P. Hirth, Phys. Status Solidi B 242 (2005) p.836-841.

[3] S.D. Gavazza and D.M. Barnett, J. Mech. Phys. Solids 24 (1976) p.171-185.

[4] J.D. Eshelby, W.T. Read and W. Shockley, Acta Metall. 1 (1953) p.251-259.

[5] A.N. Stroh, Philos. Mag. 3 (1958) p.625-646.

[6] A.N. Stroh, J. Math. Phys. (Cambridge, Mass.) 41 (1962) p.77.

[7] D.J. Bacon, D.M. Barnett and R.O. Scattergood, Prog. Mater. Sci. 23 (1980) p.51-262.

[8] W. Cai, V.V. Bulatov, J. Chang, J. Li and S. Yip, Philos. Mag. 83 (2003) p.539-567.

[9] J. Li, C.Z. Wang, J.P. Chang, W. Cai, V.V. Bulatov, K.M. Ho and S. Yip, Phys. Rev. B 70 (2004) p.104113.

[10] D.J. Bacon, R. Bullough and J.R. Willis, Philos. Mag. 22 (1970) p.31-45.

[11] T.J. Gosling and J.R. Willis, Philos. Mag. A 69 (1994) p.65-90.

[12] T.J. Gosling, Philos. Mag. A 73 (1996) p.11-45.

13] S.L. Dudarev, R. Bullough and P.M. Derlet, Phys. Rev. Lett. 100 (2008) p.135503.

[14] W. Xu and J.A. Moriarty, Phys. Rev. B 54 (1996) p.6941-6951.

[15] S. Ismail-Beigi and T.A. Arias, Phys. Rev. Lett. 84 (2000) p.1499-1502.

[16] X. Blase, K. Lin, A. Canning, S.G. Louie and D.C. Chrzan, Phys. Rev. Lett. 84 (2000) p.5780-5783.

[17] W. Cai, V. Bulatov, J. Chang, J. Li and S. Yip, Phys. Rev. Lett. 86 (2001) p.5727-5730.

[18] E. Clouet, L. Ventelon and F. Willaime, Phys. Rev. Lett. 102 (2009) p.055502.

[19] C. Teodosiu Elastic Models of Crystal Defects, Springer-Verlag, Berlin, 1982.

[20] J.P. Hirth and J. Lothe Theory of Dislocations, $2^{\text {nd }}$ Wiley, New York, 1982.

[21] J. Lothe, 1992, Vol. 31 of Modern Problems in Condensed Matter Sciencesin Elastic Strain Fields and Dislocation Mobility North-Holland, Amsterdam, p. 175.

[22] T.C.T. Ting Anisotropic Elasticity: Theory and Applications, Oxford University Press, New York, 1996.

[23] M.I. Mendelev, S. Han, D.J. Srolovitz, G.J. Ackland, D.Y. Sun and M. Asta, Philos. Mag. 83 (2003) p.3977-3994.

[24] G.J. Ackland, M.I. Mendelev, D.J. Srolovitz, S. Han and A.V. Barashev, J. Phys.: Condens. Matter 16 (2004) p.S2629-S2642.

[25] J.E. Sinclair, P.C. Gehlen, R.G. Hoagland and J.P. Hirth, J. Appl. Phys. 49 (1978) p.3890-3897.

[26] C. Woodward and S.I. Rao, Philos. Mag. A 81 (2001) p.1305-1316.

[27] C. Woodward and S.I. Rao, Phys. Rev. Lett. 88 (2002) p.216402.

[28] G. de Wit and J.S. Koehler, Phys. Rev. 116 (1959) p.1113-1120.

[29] H. Mughrabi, Mater. Sci. Eng. A 309-310 (2001) p.237-245.

[30] H. Schmid and H.O.K. Kirchner, Philos. Mag. A 58 (1988) p.905-921.

[31] G.B. Arfken and H.J. Weber Mathematical Methods for Physicists, $5^{\text {th }}$ Academic Press, San Diego, 2001. 